\title{
Effectiveness of fixed-dose combination therapy in hypertension: systematic review and meta-analysis
}

Paweł Kawalec ${ }^{1}$, Przemysław Holko ${ }^{1}$, Małgorzata Gawin ${ }^{2}$, Andrzej Pilc ${ }^{3}$

${ }^{1}$ Institute of Public Health, Faculty of Health Sciences, Jagiellonian University, Krakow, Poland

2Department of Food Biotechnology, Faculty of Food Technology, University of Agriculture, Krakow, Poland

${ }^{3}$ Institute of Pharmacology Polish Academy of Sciences, Krakow, Poland

Submitted: 24 June 2015

Accepted: 9 December 2015

Arch Med Sci 2018; 14, 5: 1125-1136

DOI: https://doi.org/10.5114/aoms.2018.77561

Copyright $\odot 2018$ Termedia \& Banach

\section{Abstract}

Introduction: Clinical studies have revealed that fixed-dose combinations (FDCs) of drugs can have a better effect on blood pressure than free-equivalent combinations (FECs). Our objectives were to perform an up-to-date assessment of the effectiveness of FDCs and FECs in antihypertensive therapy, to provide more accurate results by using a stratified meta-analysis.

Material and methods: A systematic review was performed in PubMed, Web of Science, and Cochrane databases according to PRISMA guidelines. The outcomes were adherence (compliance), persistence to medication, reduction of blood pressure and the safety profile. We used the Newcastle Ottawa scale or the Delphi list for the assessment of the quality of cohort studies or clinical trials, respectively. Heterogeneity was assessed using the Cochrane $Q$ test and $I^{2}$ statistic.

Results: Of 301 abstracts screened, 26 primary studies and 2 other metaanalyses were identified, of which 12 studies were included in the metaanalyses and 3 studies were included in the narrative review. The FDC treatment is associated with a significant improvement in adherence and persistence in comparison with FEC treatment, e.g., the average medicine possession ratio increased with FDC by $13.1 \%(p<0.001)$. For endpoints correlated with higher adherence (e.g., a reduction in blood pressure), a nonsignificant benefit was observed for FDCs. Moreover, it was demonstrated that higher adherence can lead to a lower risk of cardiovascular events.

Conclusions: In comparison with FECs, the FDC treatment is associated with a significant improvement in the cooperation between a doctor and a patient and with increased patients' adherence to the treatment schedule.

Key words: fixed-dose combinations, free-equivalent combinations, hypertension.

\section{Introduction}

Hypertension is a common condition associated with substantial morbidity and mortality. Nowadays, over 1 billion people suffer from high blood pressure worldwide [1], and it is estimated that the prevalence of hypertension will have risen by an additional 27 million people by the year 2030 [2]. Improper management of hypertension can lead to cardiac failure, stroke, end-stage renal disease, and, finally, to death [3, 4]. Although blood pressure lowering is crucial in hypertension, about half 
of the patients still have uncontrolled blood pressure [5].

The initial approach to blood pressure lowering is based on monotherapy, but in many cases, combination therapy is necessary to achieve target blood pressure levels [6]. Studies that assessed the difference in efficacy between monotherapy and polytherapy for blood pressure control have shown that combination therapy is more effective than monotherapy $[7,8]$. Combination therapy is available either as a fixed-dose combination (FDC), which includes at least 2 active agents combined in a single pill (also known as a single-pill combination), or as a free-equivalent combination (FEC, also known as a free combination), which involves separate use of the corresponding drug components. Although the FEC is chemically equivalent to the FDC, a number of studies have indicated that the FDC can have a better impact on blood pressure control as well as reducing the use of medical resources by increasing patients' adherence (compliance) and persistence to treatment [9-12].

Systematic reviews published in 2010 and 2011 confirmed a significantly higher adherence rate among patients treated with an FDC compared with an FEC [12, 13], although without a significant impact on blood pressure levels [13]. It is noteworthy that previous reviews $[12,13]$ provide different conclusions regarding persistence.

We performed a systematic review and metaanalysis to provide an up-to-date evaluation of the effectiveness of FDCs in comparison with FECs in patients with hypertension to deliver precise effect sizes calculated throughout separate meta-analyses stratified according to the definition of outcomes related to: adherence, average medicine possession ratio (MPR) or average proportion of days covered (PDC) or probability of being adherent to treatment (i.e. with MPR or PDC $\geq 80 \%$ ) or stratified according to the period of observation in case of persistence, incorporating generally accepted methodological standards (no synthesis of: prospective and retrospective studies, adjusted and unadjusted outcomes, or treating the rate of being adherent and the adherence rate as the same variable. Moreover, we aimed to provide systematically obtained evidence regarding effectiveness of FDCs in reducing rates of cardiovascular events.

We finally aimed to solve the problem of conflicting conclusions from previous reviews $[12,13]$ regarding higher persistence on FDC treatment in comparison with FEC treatment.

\section{Material and methods}

\section{Search strategy and eligibility criteria}

A systematic search of the literature was conducted in PubMed, Web of Science, and Cochrane
Controlled Trial Registry. No date restriction was applied to the search and the last search was performed on 14 April 2015. Comprehensive combinations of $\mathrm{MeSH}$ and free-text terms were used for the literature search to identify studies regarding hypertension, antihypertensive agents, and FDCs. The terms were then combined with the terms for the outcome of interest, including at least compliance, adherence, persistence, and adverse effects. The search was conducted according to the Preferred Reporting Items for Systematic Reviews and MetaAnalyses (PRISMA) guidelines for systematic literature reviews and meta-analyses [14]. Potentially relevant full-text publications were then screened for inclusion against the following predetermined criteria: patients with hypertension (population); fixed-dose combinations with or without additional drugs (intervention) compared with free-equivalent combinations with or without additional drugs (comparison); medication compliance/adherence, persistence, adverse events, systolic and diastolic arterial blood pressures, and safety of treatment as outcomes. No restrictions were applied as to the study design. The study was excluded if it did not comply with at least one inclusion criterion.

Within the framework of our systematic review, apart from primary research publications identified during the database search, we also took into consideration publications and conclusions presented in previously published systematic reviews $[12,13]$.

Records selected for retrieval were assessed by 2 independent reviewers for methodological validity before inclusion in the review using standardized critical appraisal instruments. Any disagreements were resolved through discussion or with the third independent reviewer.

Quality assessment of the included studies was done using either the Newcastle-Ottawa scale (cohort studies) [15] or the Delphi list (clinical trials) [16], and the studies were accordingly categorized into the following 4 categories: poor, fair, good, and excellent.

\section{Data collection and synthesis}

Quantitative data were extracted from papers included in the review using the standardized data extraction tables. The extracted data included specific details about the interventions, patients' characteristics, study design, methods of outcome analysis, and results of significance to the review questions and specific objectives. Data extraction and meta-analysis were conducted independently on the basis of all the identified studies providing an update and verification of the systematic reviews $[12,13]$.

Medication adherence (compliance) was defined according to: i) the number of the administered tablets as a medicine possession ratio (MPR) 
estimated on the basis of the number of days of medication supply within the prescription refill interval [17-26] or ii) the percentage of days during which a medication was taken by patients, on the basis of the proportion of days covered (PDC) [27, 28]. A compliant (adherent) patient was defined as a patient with an MPR or PDC of $80 \%$ or higher. Persistence was described on the basis of the prescription refill interval as previously described by Gupta et al. [13].

We investigated the average estimate of adherence estimators (PDC or MPR), probability of being adherent to the prescribed treatment (MPR or PDC $\geq 80 \%$ ), effectiveness in blood pressure lowering, as well as persistence rates and the rates of adverse events and cardiovascular events among all patients treated with an FDC or FEC. The publication bias was assessed by funnel plots and by Egger's or Begg's test. Effect sizes were expressed as odds ratios (ORs) for dichotomous data and weighted mean differences for continuous data. The $95 \%$ confidence intervals (Cls) were calculated for all measures. The results for time-to-event data were presented as a hazard ratio (if available) or as the average or median length of treatment calculated with nonparametric or semiparametric approaches (Kaplan-Meier or Cox model).

Standard methods were used for meta-analysis (Mantel-Haenszel fixed effect model and DerSimonian and Laird random effect model). Heterogeneity was assessed using the standard $\chi^{2}$ (Cochrane Q) test and $l^{2}$ statistic. The random effects model was applied if significant heterogeneity between studies was revealed (a $p$-value of less than 0.1 ). Heterogeneity between studies was explored using subgroup analyses based on different quantitative study designs included in this review (prospective vs. retrospective), different measure of adherence (MPR vs. PDC), and different follow-up periods for dichotomous outcomes. The meta-regression for outcomes reported in more than 5 studies was also planned as an attempt to explain the heterogeneity. Where statistical pooling was not possible, the findings were presented in a narrative form including tables to enhance data presentation where appropriate.

All calculations were performed using the software R version 3.03 (R Core Team, 2014) and Excel 2013 (Microsoft). The 'metafor' package was used within the R environment [29].

\section{Results}

The electronic searches yielded 301 unique records after duplicates had been removed. The selection of titles and abstracts resulted in 34 potentially relevant articles (32 primary research studies and 2 systematic reviews), of which 6 articles were excluded (lack of appropriate data to use, 1; analysis of other endpoints, 2; inadequate comparison, 3). The flow of information through the different phases of the systematic review is shown in Figure 1.

In a systematic review by Gupta et al. [13], conducted in 2010, 15 studies were taken into account: 5 randomized studies [17, 30-33] (1 parallel trial and 4 crossover trials, 2 double-blinded and 3 open trials), 4 nonrandomized crossover trials [ 18 , 34-36], and 6 retrospective cohort studies [19-21, $37,38]$ (a study by Dezii, 2000, presented data for 2 different treatment schedules, FDC vs. FEC; therefore, it was introduced separately).

In a systematic review by Sherill et al. [12], 15 studies were taken into account: 1 randomized crossover multicenter study [17], 1 nonrandomized crossover multicenter study [18], and 13 retrospective cohort studies [19-26, 37, 39-42].

Six studies [17-21, 37] included in the review by Sherrill et al. [12] were also investigated by Gupta et al. [13]. Two studies (Malesker and Hilleman, 2010 [42], and Barron et al., 2008 [41]), which were included by Sherrill et al. [12], were excluded from our analysis because they focused on the analysis of other endpoints. Two bibliographic records [22, 23] identified by Sherrill et al. [12] were related to the studies included by Gupta et al. [13].

In our analysis a conference abstract (Jackson et al., 2006 [38]) included in the review by Gupta et al. [13] was replaced by a full-text publication (Brixner et al. 2008 [22]), which concerned the same study although with slight methodological changes in data analysis. A study performed by Dickson and Plauschinat, 2008 [23], included data collected in the population of people between the ages of 18 and 99 years old, while another study by Dickson and Plauschinat, 2008 [21], which was included in the review by Gupta et al., 2010

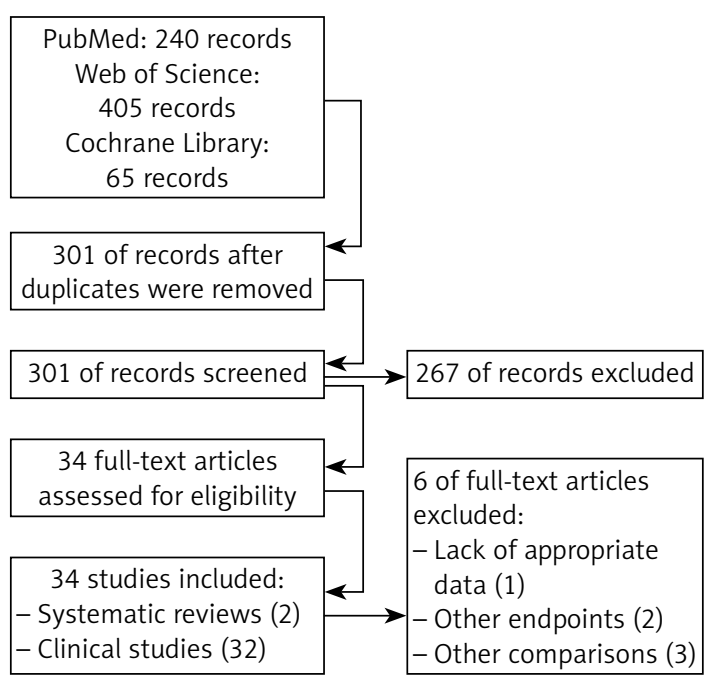

Figure 1. PRISMA flow diagram for selection of studies identified in the systematic review 
[13], contained data collected in the population of people between the age of 65 and 99 years old (2 samples from the same study's population).

Six new retrospective studies were identified [27, 28, 40, 43-45], but only four met the criteria for inclusion in the analysis. Studies by Asplund et al. 1984 [17], Kumagai et al. 2013 [44], and Ulusoy et al. 2012 [45] were not included in statistical pooling because the data reported did not allow for their use in the review or the clinical assessment was different in those studies.

Finally, in our analysis we included data from four studies [18-21], which were also taken into account by Gupta et al. and Sherrill et al., from 5 studies [2-14, 17-26, 30-39] which were included only by Sherill et al. and from 6 new identified studies [3, 27, 28, 43, 44].

Characteristics of the identified studies [17-28, 30-40, 43-45] are shown in Table I.

\section{Adherence}

In our analysis 11 retrospective cohort studies and 1 nonrandomized trial reporting data on adherence to medication in hypertensive patients were taken into account (studies providing information on the average MPR or PDC or the number of adherent patients in each group).

A meta-analysis of the 2 retrospective cohort studies that defined being adherent as having an MPR of 0.8 or higher demonstrated a significantly $(p<0.001)$ higher percentage of adherent patients on FDC treatment in comparison with those on FEC treatment (pooled OR at 1.47; 95\% Cl: 1.23-1.74). Similar results were obtained in a meta-analysis of the 2 retrospective cohort studies with the PDC as the measure of adherence, although with significant heterogeneity of effect sizes ( $p<0.001)$. A significant increase in the rate of adherence on FDC treatment was also obtained in 1 prospective clinical trial ( $p=0.032$; Figure 2$)$.

Nine retrospective cohort studies showed the results for the average MPR (4 studies reported variances and 4 did not have variances available) or PDC values (1 study). A meta-analysis of 4 cohort studies demonstrated that using the FDC instead of the FEC was associated with a significant increase in the average MPR by absolute $13.1 \%$ (95\% Cls: $8.9-17.2 \%, p<0.001$ ). A significant increase in the average PDC was also reported by Ferrario et al. ( $p<0.001$; Figure 3) [28].

\section{Persistence}

Data concerning persistence were included in the 6 identified studies. One of them [37] was previously identified by Gupta et al. [13], and four $[22,24,26,37]$ were previously described by Sherrill et al. [12]. The sixth study was identified in our search. A study by Hasford et al. 2007, in- cluded by Sherrill et al. [12], was excluded from the meta-analysis due to different definitions of FDC and FEC in the compared groups [39]. A metaanalysis performed only on the basis of retrospective cohort studies showed significantly higher persistence among patients treated with the FDC when compared with those treated with the FEC. After 6 and 12 months of treatment, the number of patients on FDC treatment was significantly higher than that of those on FEC treatment ( $p=0.024$ and $p=0.012$, respectively; Figure 4). A significant increase in the duration of treatment was also identified ( $p<0.001$; Table II).

\section{Efficacy in blood pressure lowering}

Nine trials included in the systematic review by Gupta et al. [13] provided information on blood pressure-lowering efficacy, and additionally, three of those trials reported the influence on both systolic and diastolic blood pressures.

Apart from the studies found by Gupta [13] and described in his systematic review, we identified three additional clinical trials [43-45] referring to blood pressure-lowering efficacy that were included in the narrative review - we evaluated the new trials and established that they are not eligible to be included in the updated meta-analysis.

In a cohort study performed by Bronsert et al. [43], patients receiving FDC treatment in comparison with those receiving FEC treatment showed a significantly greater reduction in systolic blood pressure ( $-5.3 \mathrm{~mm} \mathrm{Hg} ; 95 \% \mathrm{Cl}:-6.5$ to -4.1$)$ and diastolic blood pressure $(-4.1 \mathrm{~mm} \mathrm{Hg}$; $95 \% \mathrm{Cl}:-4.8$ to -3.4). The analysis was adjusted for the difference in confounder variables between the compared groups. A significantly higher rate of blood pressure normalization on FDC treatment in comparison with FEC treatment was also found $(p<0.001)$ [43]. Kumagai et al. [44] found that home blood pressure obtained by treatment was significantly lower in patients receiving FDCs than those receiving FECs, and this was accompanied by increasing adherence to medication $(p<0.01)$. On the other hand, Ulusoy et al. [45] demonstrated a reduction in all-day, daytime, night-time, and early morning systolic and diastolic blood pressures (blood pressure variability) in the FEC group as compared with the FDC group. However, the conclusions should probably be attributed to the difference in the time of administration of FEC compounds in the control group (valsartan in the morning and amlodipine in the afternoon in the control group; FDC in the morning in the study group) [45].

\section{Other health outcomes and adverse events}

Unfortunately, as in the case above, we have not identified new data on adverse effects, which 


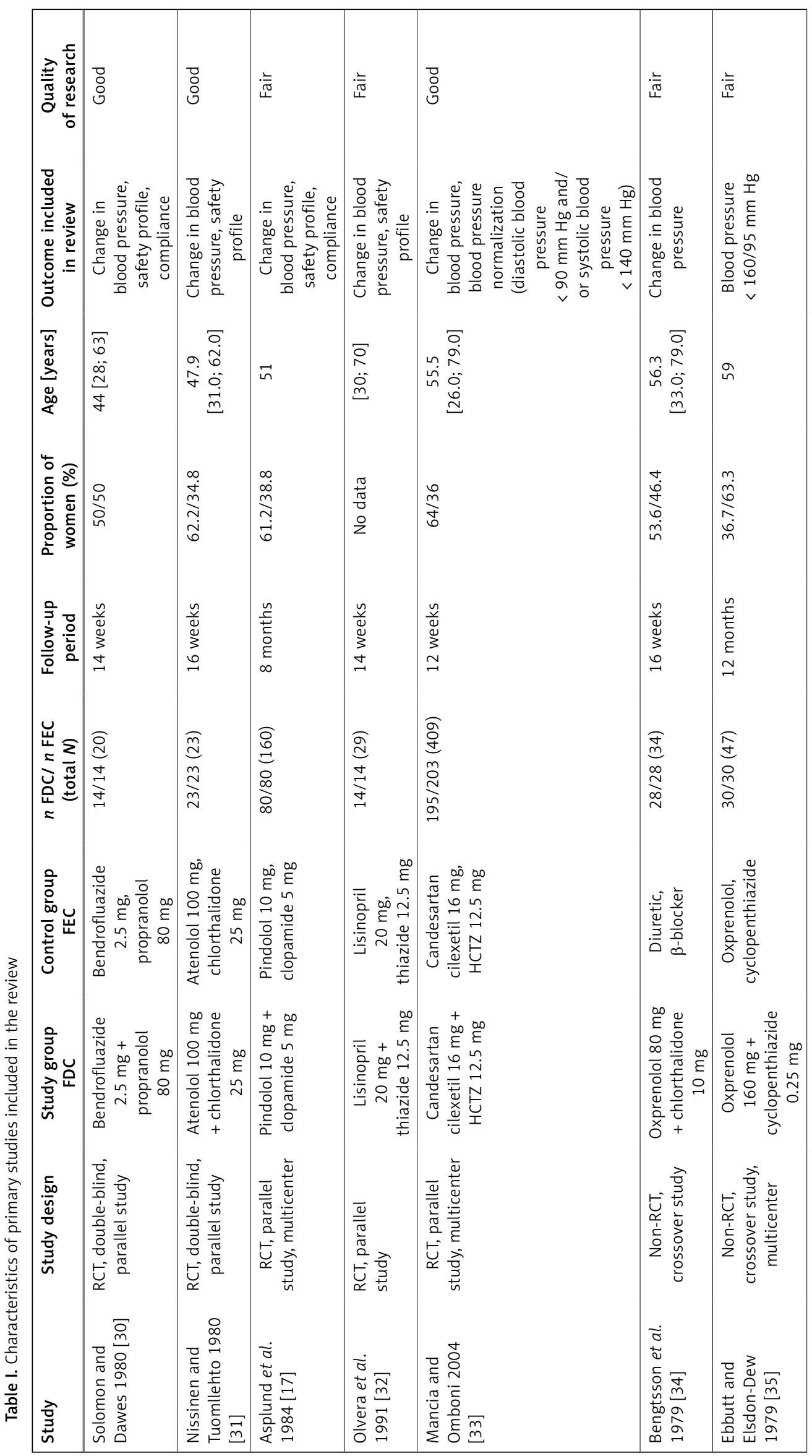




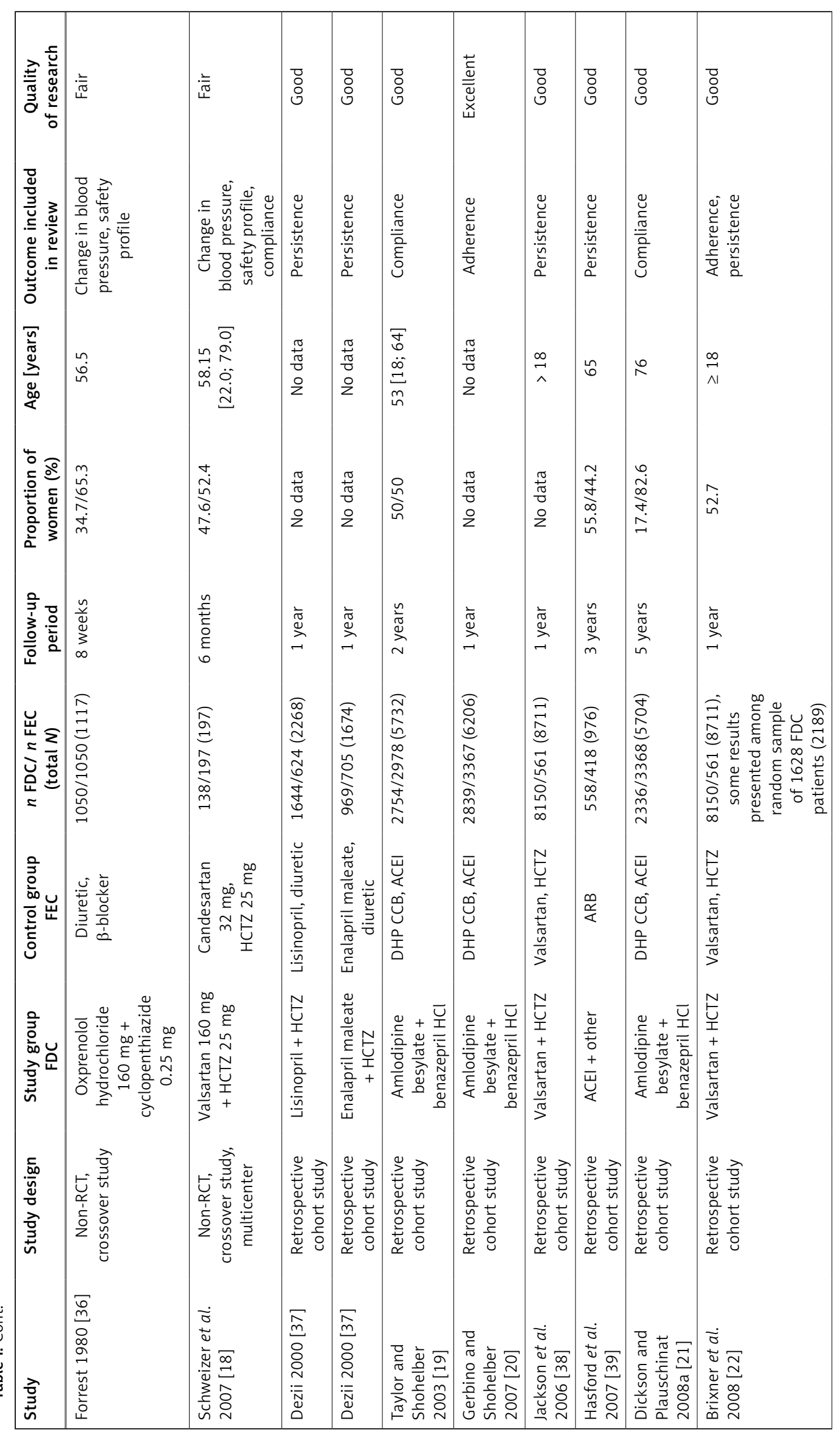




\begin{tabular}{|c|c|c|c|c|c|c|c|c|c|c|}
\hline 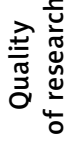 & $\begin{array}{l}\text { D } \\
8 \\
0\end{array}$ & $\begin{array}{l}\text { ¿ } \\
\text { ০ }\end{array}$ & 言 & $\begin{array}{l}0 \\
0 \\
0 \\
0\end{array}$ & $\begin{array}{l}\text { : } \\
\stackrel{\bar{\sigma}}{\longleftarrow}\end{array}$ & $\begin{array}{l}\bar{x} \\
\circ \\
0\end{array}$ & 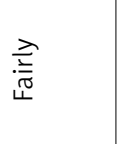 & $\begin{array}{l}\text { zo } \\
\text { o }\end{array}$ & $\begin{array}{l}\text { Do } \\
0 \\
0\end{array}$ & 言 \\
\hline 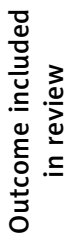 & 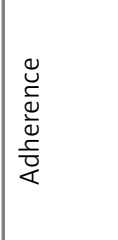 & 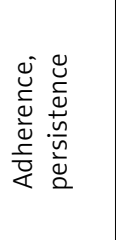 &  & 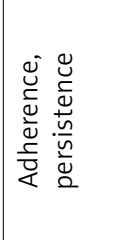 & 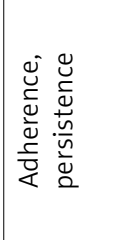 & 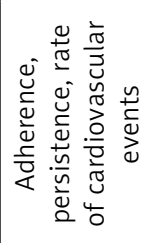 & 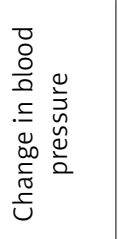 & 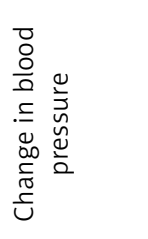 & 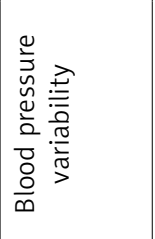 &  \\
\hline 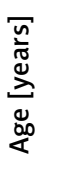 & 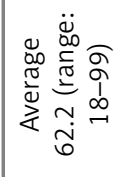 & 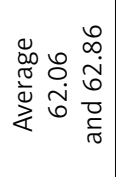 & $\stackrel{\infty}{\stackrel{\infty}{\wedge}}$ &  & 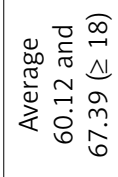 & 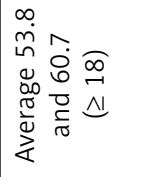 &  & 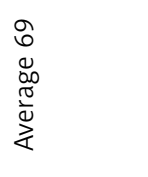 & 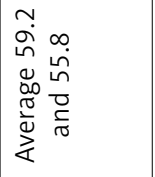 & $\stackrel{\infty}{\stackrel{\infty}{\wedge}}$ \\
\hline 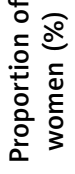 & $\hat{\dot{I}}$ & $\begin{array}{l}a \\
\text { iे } \\
\text { in }\end{array}$ & $\begin{array}{l}n \\
\tilde{n} \\
\tilde{b}\end{array}$ & $\begin{array}{l}0 \\
\dot{n} \\
i n\end{array}$ & \begin{tabular}{|l}
0 \\
0 \\
0 \\
1 \\
$m$ \\
$\dot{f}$ \\
$\dot{f}$
\end{tabular} & $\begin{array}{l}\stackrel{0}{\dot{b}} \\
\dot{q}\end{array}$ & 吕 & 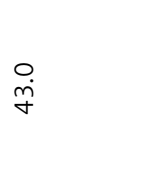 & $\vec{i}$ & 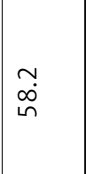 \\
\hline 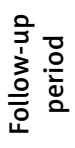 & $\mid \begin{array}{l}\overline{\bar{\sigma}} \\
\stackrel{\bar{\sigma}}{-} \\
-1\end{array}$ & 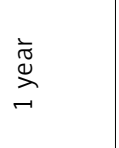 & 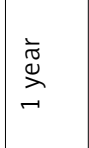 &  & 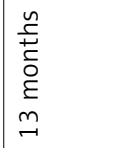 & 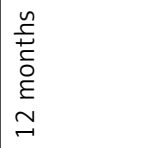 & 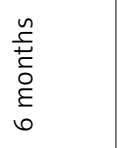 & 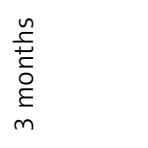 & 1 & 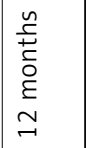 \\
\hline 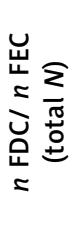 & $\begin{array}{l}0 \\
0 \\
0 \\
0 \\
+ \\
m \\
1 \\
\hat{n} \\
0 \\
0 \\
m \\
m\end{array}$ & 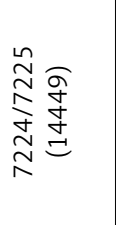 & 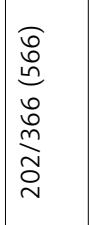 & 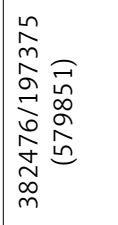 & 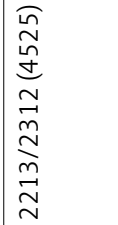 & 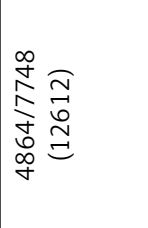 & 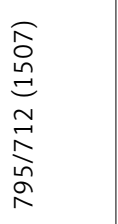 & 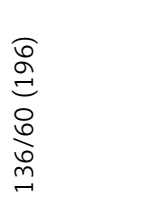 & 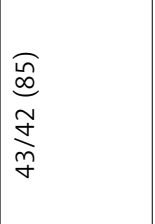 & 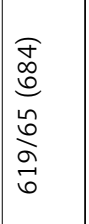 \\
\hline 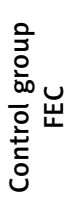 & 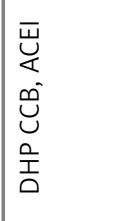 & 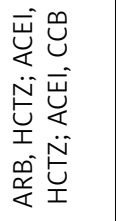 & 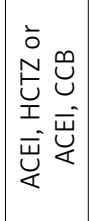 & 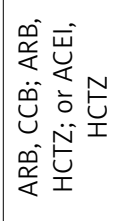 & 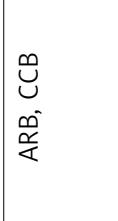 & 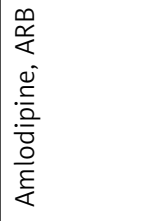 & 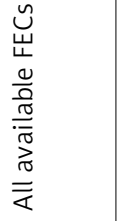 & 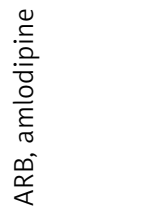 & 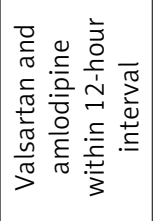 & 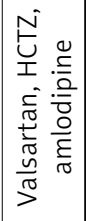 \\
\hline 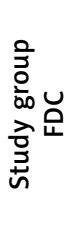 & 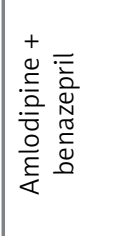 &  & 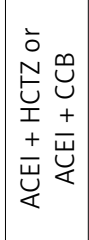 & 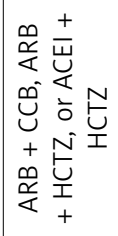 & 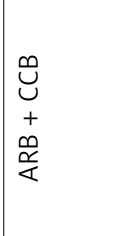 & 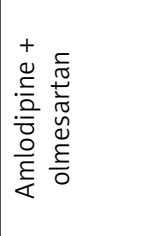 & 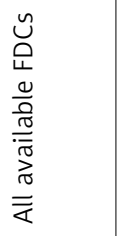 & 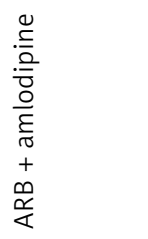 & 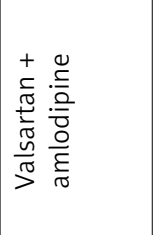 & 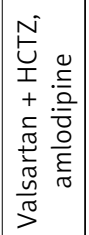 \\
\hline 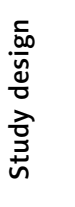 & 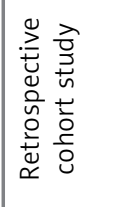 & 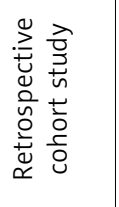 & 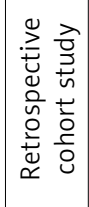 & 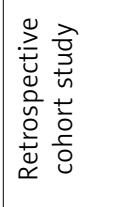 & 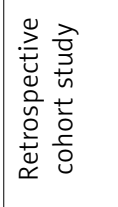 & 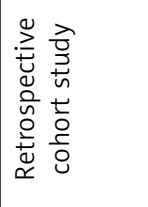 & 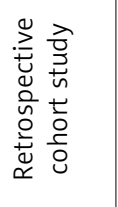 & 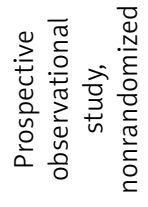 & 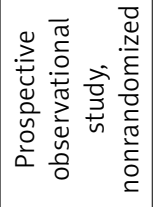 &  \\
\hline 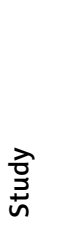 & 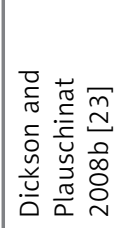 & 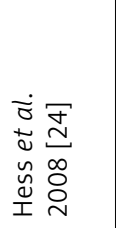 & 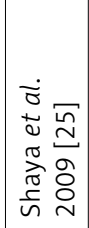 & 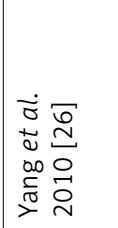 & 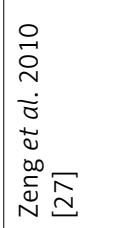 & 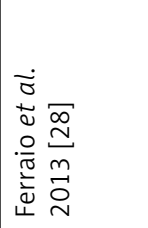 & 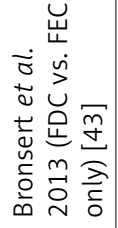 & 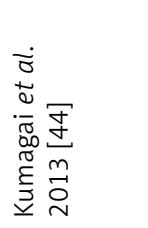 & 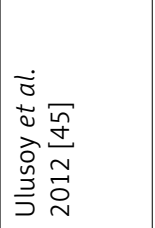 & 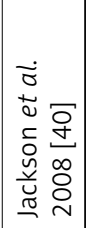 \\
\hline
\end{tabular}




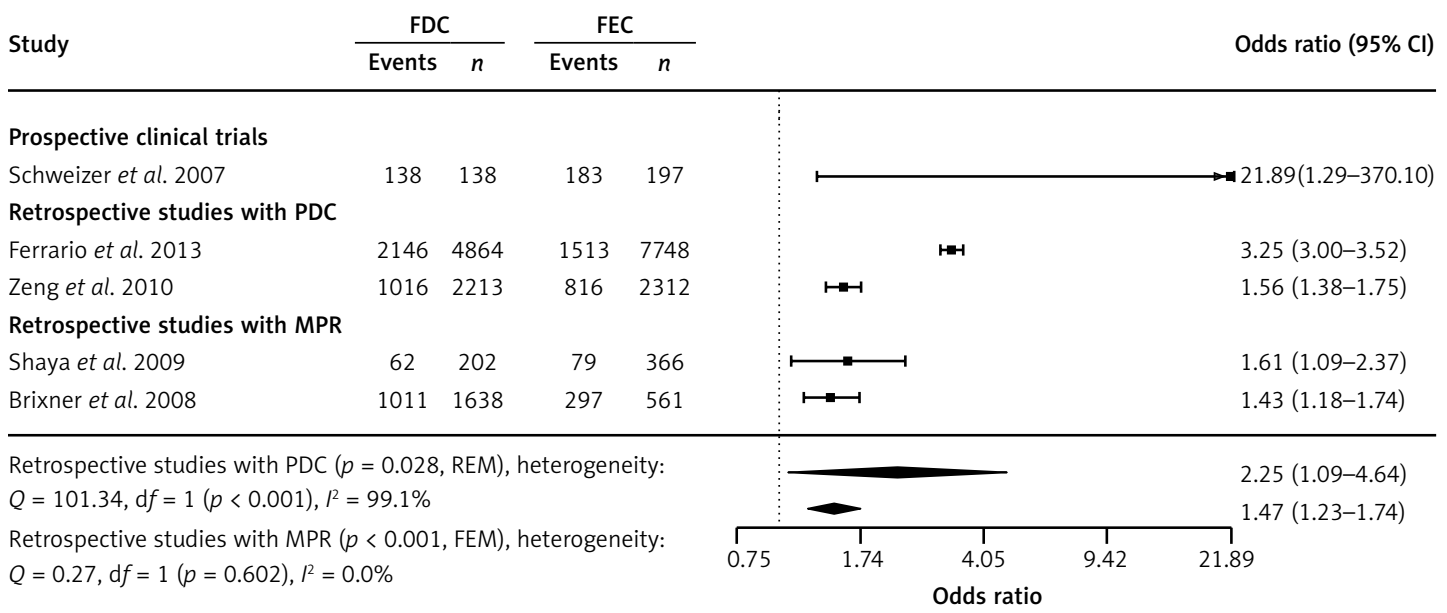

Figure 2. Meta-analysis of odds ratio for adherence to treatment. Events represent the number of patients meeting the definition of adherence (MPR or PDC $\geq 0.8$ ). Odds ratio $(O R)$ presented for FDC in comparison with FEC, with $95 \%$ confidence intervals $(\mathrm{Cl})$

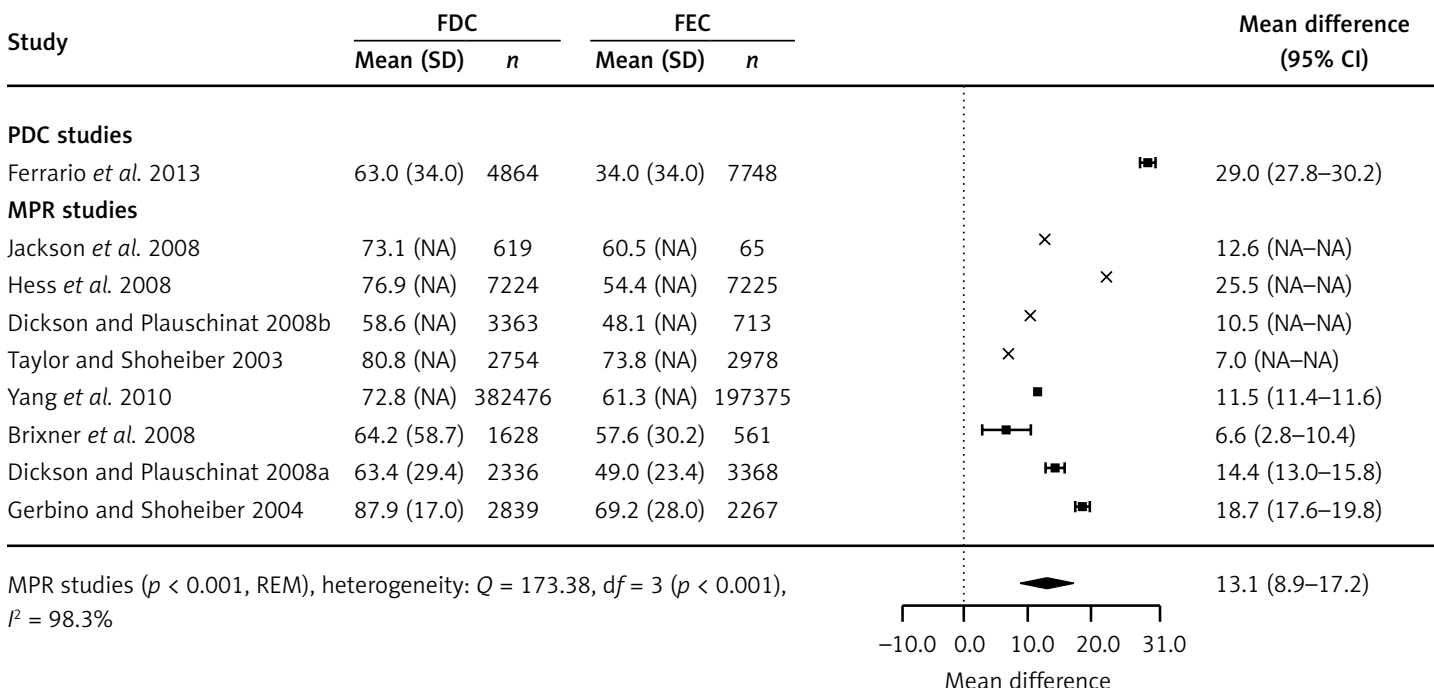

Figure 3. Meta-analysis of weighted mean difference in MPR or PDC. Mean difference presented for FDC in comparison with $\mathrm{FEC}$, with $95 \%$ confidence intervals $(\mathrm{Cl})$. Studies not reporting variance for outcomes marked by cross estimates

NA - not available.

could be incorporated into the meta-analysis. However, Ferrario et al. 2013 [28] reported the results regarding the prevalence of cardiovascular events. The analysis of this retrospective cohort study demonstrated that a significantly lower number of patients on FDC treatment experienced cardiovascular events compared with patients on FEC treatment $(\mathrm{OR}=0.31 ; 95 \% \mathrm{Cl}: 0.24-0.39)$.

\section{Bias assessment and heterogeneity}

Significant heterogeneity was observed in relation to all of the endpoints included in the review when all studies were pooled (data not shown). Subgroup analyses reduced the heterogeneity for some outcomes (odds for being adherent to treatment as measured with MPR in cohort stud- ies, differences in average MPR between cohort studies), while for others heterogeneity remained significant.

A series of univariate and multivariate meta-regression analyses for 2 endpoints was carried out (adherence rates and average measure of adherence; 12 studies). The following predictors were taken into account: type of the study (prospective vs. retrospective), measure of adherence (PDC vs. $M P R$ ), duration of follow-up, percentage of women, and mean age of study participants. Based on the results obtained from meta-regression analyses, there was no significant effect of any of the predictors (data not shown).

The publication bias was not identified, although a reliable conclusion cannot be drawn 


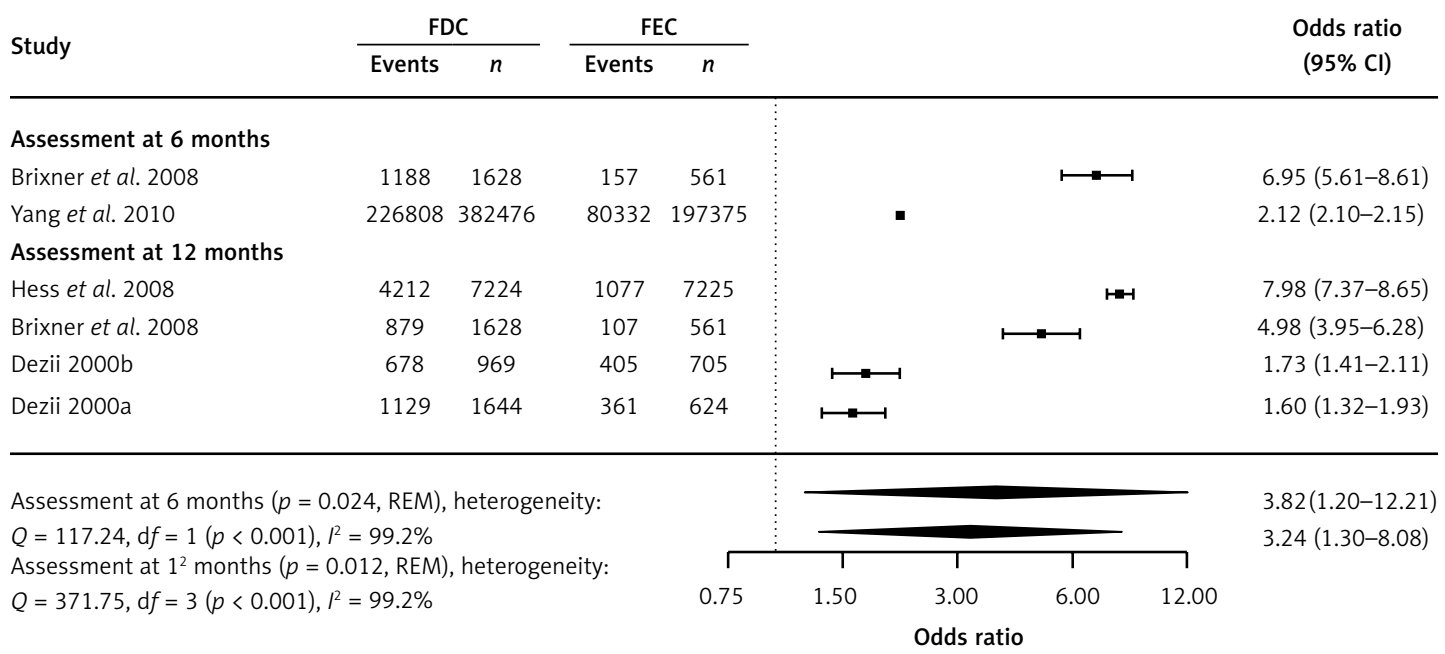

Figure 4. Meta-analysis of persistence. Events represent the number of patients meeting the definition of persistence. Odds ratio (OR) presented for FDC in comparison with FEC, with 95\% confidence intervals (CI)

Table II. Clinical efficacy (continuous endpoints) of fixed-dose combinations (FDC) compared with free-equivalent combinations (FECS): persistence defined as average duration of treatment

\begin{tabular}{|c|c|c|c|c|}
\hline Study & $\begin{array}{l}\text { FDC group } \\
\text { Mean } \pm \text { SD } \\
(N)\end{array}$ & $\begin{array}{l}\text { FEC group } \\
\text { Mean } \pm \text { SD } \\
(N)\end{array}$ & $\begin{array}{l}\text { Effect size } \\
(95 \% \mathrm{Cl})\end{array}$ & $P$-value \\
\hline Zeng et al. 2010 [35] & $\begin{array}{c}215.56 \pm \text { NA } \\
(2213)\end{array}$ & $\begin{array}{c}174.48 \pm N A \\
(2312)\end{array}$ & $\begin{array}{l}\mathrm{HR}=0.66 \\
(0.63-0.70)\end{array}$ & $<0.001$ \\
\hline $\begin{array}{l}\text { Ferrario et al. } \\
2013[36]\end{array}$ & $\begin{array}{c}339.76 \pm 224.08 \\
(2146)\end{array}$ & $\begin{array}{c}233.72 \pm 238.56 \\
(1513)\end{array}$ & $\begin{array}{c}M D=106.04 \\
(90.90-121.18)\end{array}$ & $<0.001$ \\
\hline
\end{tabular}

$H R$ - hazard ratio, $M D$ - mean difference, $N A$ - not available.

owing to the small number of studies. Neither the funnel plots nor Egger's test indicated any association between the effect size and study size.

\section{Discussion}

A systematic review with a meta-analysis and narrative synthesis was conducted to compare the effectiveness of the FDC and FEC with regards to adherence (compliance) to medication, persistence to medication, and other clinically important endpoints during treatment of arterial hypertension. The results of the comparison can be important for the choice of treatment because the majority of patients with hypertension need more than 1 drug to control blood pressure.

Based on the synthesis of direct-comparison studies, it was found that FDC treatment is associated with a significant improvement in adherence and persistence in comparison with FEC treatment. It can be argued that the increase in adherence and persistence to FDC treatment in comparison with FEC treatment should result in a greater reduction in blood pressure and thus in obtaining lower blood pressure values. We did not identify any clear evidence to confirm this effect. The evidence for the benefit of FDCs over FECs regarding the blood pressure-lowering effect was sparse and indicated only in large cohort studies. However, we found direct evidence for the reduction in the rate of cardiovascular events on FDC treatment.

The reduction in the rate of cardiovascular events can be indirectly confirmed by the results of 2 studies indicating that patients adherent to antihypertensive treatment are experiencing minor cardiovascular events. In a study that included patients with hypertension and without cardiovascular disease at baseline, a reduction in the annual risk of cardiovascular events by $22 \%(95 \% \mathrm{Cl}$ : 13-30\%) was observed among adherent compared with nonadherent patients [46]. Similarly, a study that included patients with hypertension and without prior stroke reported a reduction in the risk of stroke and death by $8 \%(95 \% \mathrm{Cl}: 4-13 \%)$ and $7 \%$ (95\% Cl: 4-10\%), respectively, among adherent versus nonadherent patients [47].

In general, our results are in line with the previously published systematic reviews by Sherrill et al. 2011 [12] and Gupta et al. 2010 [13], although some changes were observed, especially when comparing the conclusions with the latter study. 
Sherrill et al. 2011 [12] demonstrated significantly higher adherence to treatment in the FDC group compared with the FEC group, based on a meta-analysis of studies reporting average MPR in the groups (13.31\%, 95\% Cl: 8.26-18.35\%).

Likewise, Gupta et al. [13] revealed that the adherence ratio in the FDC group was 1.2 times higher than that in the FEC group. However, they found that an increase in adherence was not statistically significant according to the pooled results of prospective trials, had borderline significance according to the pooled results of cohort studies, and was statistically significant according to the pooled results of all studies - prospective and retrospective combined [13].

Similarly, Bangalore et al. 2007 [48] found that FDC treatment decreased the risk of medication noncompliance in patients with hypertension, tuberculosis, diabetes, and human immunodeficiency virus.

Considering patient's persistence measured by the prescription refill interval and as duration of treatment, a significant advantage of FDC over FEC was identified. This is in line with the results reported by Sherrill et al. 2011 [12] (pooled risk ratio for 5 studies at $2.13 ; 95 \% \mathrm{Cl}: 1.11-4.09$ ), but not with those reported by Gupta et al., 2010 [13], who found no statistically significant differences between the FDC and FEC groups (pooled OR for 3 studies at 1.54; 95\% Cl: 0.95-2.49). These discrepancies might have been caused by the inclusion of a greater number of studies in our analysis compared with the analysis by Gupta et al. [13], the replacement of data from the conference abstract (Jackson et al. 2006 [38]) by the full-text publication (Brixner et al. 2008 [22]), or by the bias in data extraction or analysis (effect size estimation and description) in the meta-analysis previously published by Gupta et al. [13]. Interestingly, Hasford et al. 2007 demonstrated that persistence was the longest for FDCs of angiotensin receptor blockers than for FDCs of other drug classes. After 3 years of follow-up, $17.7 \%$ of the patients in the FDC group and only $3.2 \%$ of those in the FEC group were still on the initial treatment, although differences in active substances used in both arms were identified [39].

The meta-analysis by Gupta et al. showed a nonsignificant decline in the rates of adverse effects associated with the use of the FDC as compared with the use of the FEC $(O R=0.80 ; 95 \% \mathrm{Cl}$ : 0.58-1.11) [13]. We have found no new evidence on the safety profile, so the results presented by Gupta et al. 2010 [13] seem to be currently valid, and therefore we did not perform a meta-analysis regarding this endpoint. Only 1 retrospective study provided evidence concerning the incidence of cardiovascular events in the compared groups. The results for blood pressure and cardiovascular events should be interpreted with caution owing to the absence of other studies reporting data on these outcomes. We relied on the results of retrospective nonrandomized studies, which by definition have less credibility than prospective randomized clinical trials. On the other hand, reliable randomized clinical trials will be difficult to conduct for adherence-based therapies.

As for the reduction in systolic and diastolic blood pressures and normalization of blood pressure, no new study that could expand the metaanalysis by Gupta et al. 2010 [13] was identified. Instead, 2 large retrospective cohort studies by Bronsert et al., 2013 [43], and Kumagai et al., 2013 [44], were found. Both studies indicated that blood pressure was significantly lower in patients receiving FDC treatment than in those receiving FEC treatment. However it should be noted that Gupta et al. [13] found a higher but not statistically significant $(p>0.05)$ difference between the FDC and FEC in the reduction of both systolic and diastolic arterial blood pressures $(-4.1 \mathrm{~mm} \mathrm{Hg}$; 95\% Cl: -9.8 to 1.5 and $-3.1 \mathrm{~mm} \mathrm{Hg} ; 95 \% \mathrm{Cl}:-7.1$ to 0.9 , respectively). The probability of normalization of blood pressure was also comparable $(p>0.05)$ in both groups [13].

Although some contradictory conclusions were obtained by Ulusoy et al. [45] (lower blood pressure variability during FEC treatment than during FDC treatment, which could result in a reduced rate of cardiovascular events), the finding on the advantage of FDC over FEC regarding cardiovascular events diminished the significance of that effect.

In general, the major limitation of our review is the fact that the conclusions were mainly based on the results of retrospective cohort studies owing to the lack of adequate randomized trials. Moreover, the studies were characterized by high heterogeneity in terms of methodology and clinical approach. The absence of conformity for possible disturbing factors in the event of some observational studies and nonrandomized trials in our meta-analysis is another limitation. Similarly, the included studies did not take into account the presence of comorbidities and concomitant therapies. All these limitations may have affected our results. However, we did not aggregate results of studies with significant differences in design and outcomes, thus providing reliable estimates of effect sizes.

In conclusion, this systematic review besides updating the previous results for comparison of the effectiveness of FDCs and FECs in hypertensive patients also provides more accurate results through the use of a separate meta-analysis stratified in view of the definition of outcomes related to adherence or period of observation in case of persistence and additionally assessments of the 
impact of FDCs on incidence of cardiovascular events.

Our analysis demonstrated that administration of the FDC for antihypertensive treatment is associated with a statistically significant improvement in the cooperation between a doctor and a patient in comparison with FECs (increased adherence and persistence to the treatment schedule). It was shown that the use of FDCs of hypertensive agents may have a positive effect on blood pressure and is associated with a lower risk of cardiovascular events compared with the use of FECs, although clear evidence was sparse.

Well-designed randomized trials are needed to confirm our findings and to present a direct quantitative link between an increase in adherence or persistence to medication and clinically relevant outcomes such as the rate of cardiovascular events.

The introduction of FDC drugs to the standard treatment of hypertension could improve the quality of life and even provide additional benefits such as a reduced risk of cardiovascular events due to elevated adherence and persistence to the treatment.

\section{Conflict of interest}

The authors declare no conflict of interest.

\section{References}

1. Kearney PM, Whelton M, Reynolds K, et al. Global burden of hypertension: analysis of worldwide data. Lancet 2005; 365: 217-23.

2. Heidenreich P, Trogdon J, Khavjou O, et al. Forecasting the future of cardiovascular disease in the United States: a policy statement from the American Heart Association. Circulation 2011; 123: 933-44.

3. Psaty B, Furberg C, Kuller L, et al. Association between blood pressure level and the risk of myocardial infarction, stroke, and total mortality: the Cardiovascular Health Study. Arch Intern Med 2001; 161: 1183-92.

4. Chobanian AV, Bakris GL, Black HR, et al. Seventh Report of the Joint National Committee on Prevention, Detection, Evaluation, and Treatment of High Blood Pressure: The JNC 7 Report. JAMA 2003; 289: 2560-72.

5. Roger VL, Go AS, Lloyd-Jones DM, et al. Executive summary: heart disease and stroke statistics-2012 update: a report from the American Heart Association. Circulation 2012; 125: 188-97.

6. Black H, Elliott W, Neaton J, et al. Baseline characteristics and early blood pressure control in the CONVINCE trial. Hypertension 2001; 37: 12-8.

7. Brown MJ, McInnes GT, Papst CC, Zhang J, Mac-Donald TM. Aliskiren and the calcium channel blocker amlodipine combination as an initial treatment strategy for hypertension control (ACCELERATE): a randomised, parallel-group trial. Lancet 2011; 377: 312-20.

8. Neutel JM, Mancia G, Black HR, et al.; TEAMSTA Severe HTN Study Investigators. Single-pill combination of telmisartan/amlodipine in patients with severe hypertension: results from the TEAMSTA Severe HTN Study. J Clin Hypertens 2012; 14: 206-15.
9. Gerbino PP, Shoheiber O. Adherence patterns among patients treated with fixed-dose combination versus separate antihypertensive agents. Am J Health Syst Pharm 2007; 64: 1279-83.

10. Schroeder K, Fahey T, Ebrahim S. How can we improve adherence to blood pressure-lowering medication in ambulatory care? Systematic review of randomized controlled trials. Arch Intern Med 2004; 164: 722-32.

11. Baser O, Andrews LM, Wang L, et al. Comparison of realworld adherence, healthcare resource utilization and costs for newly initiated valsartan/amlodipine singe-pill combination versus angiotensin receptor blocker/calcium channel blocker free-combination therapy. J Med Econ 2011; 14: 576-83.

12. Sherrill B, Halpern M, Khan S, Zhang J, Panjabi S. Single-pill vs. free-equivalent combination therapies for hypertension: a meta-analysis of health care costs and adherence. J Clin Hypertens 2011; 13: 898-909.

13. Gupta AK, Arshad S, Poulter NR. Compliance, safety, and effectiveness of fixed-dose combinations of antihypertensive agents: a meta-analysis. Hypertension 2010; 55 : 399-407.

14. Moher D, Liberati A, Tetzlaff J, Altman DG; The PRISMA Group. Preferred Reporting Items for Systematic Reviews and Meta-Analyses: The PRISMA Statement. PLoS Med 2009; 6: e1000097.

15. Wells GA, Shea B, O'Connell D, et al. The Newcastle-Ottawa Scale (NOS) for assessing the quality of nonrandomised studies in meta-analyses. Ottawa.

16. Verhagen A, de Vet H, de Brie R, et al. The Delphi list: a criteria list for quality assessment of randomized clinical trials for conducting systematic reviews developed by Delphi consensus. J Clin Epidemiol 1998; 51: 1235-41.

17. Asplund J, Danielson M, Ohman P. Patients compliance in hypertension - the importance of number of tablets. Br J Clin Pharmacol 1984; 17: 547-52.

18. Schweizer J, Hilsmann U, Neumann G, Handrock R, Klebs S. Efficacy and safety of valsartan 160/HCTZ 25 $\mathrm{mg}$ in fixed combination in hypertensive patients not controlled by candesartan $32 \mathrm{mg}$ plus HCTZ $25 \mathrm{mg}$ in free combination. Curr Med Res Opin 2007; 23: 2877-85.

19. Taylor AA, Shoheiber O. Adherence to antihypertensive therapy with fixed-dose amlodipine besylate/benazepril $\mathrm{HCl}$ versus comparable component-based therapy. Congest Heart Fail 2003; 9: 324-32.

20. Gerbino PP, Shoheiber O. Adherence patterns among patients treated with fixed-dose combination versus separate antihypertensive agents. Am J Health Syst Pharm 2007; 64: 1279-83.

21. Dickson M, Plauschinat CA. Compliance with antihypertensive therapy in the elderly: a comparison of fixeddose combination amlodipine/ benazepril versus component-based free-combination therapy. Am J Cardiovasc Drugs 2008; 8: 45-50.

22. Brixner DI, Jackson KC, Sheng X, et al. Assessment of adherence, persistence, and costs among valsartan and hydrochlorothiazide retrospective cohorts in free-and fixed-dose combinations. Curr Med Res Opin 2008; 24: 2597-607.

23. Dickson M, Plauschinat CA. Racial differences in medication compliance and healthcare utilization among hypertensive Medicaid recipients: fixed-dose vs free-combination treatment. Ethn Dis 2008; 18: 204-9.

24. Hess G. Medication utilization patterns and hypertension-related expenditures among patients who were switched from fixed-dose to free-combination antihypertensive therapy. PT 2008; 33: 652-66. 
25. Shaya FT, Du D, Gbarayor CM, et al. Predictors of compliance with antihypertensive therapy in a high-risk Medicaid population. J Natl Med Assoc 2009; 101: 34-9.

26. Yang W, Chang J, Kahler KH, et al. Evaluation of compliance and health care utilization in patients treated with single pill vs. free combination antihypertensives. Curr Med Res Opin 2010; 26: 2065-76.

27. Zeng F, Patel BV, Andrews L, et al. Adherence and persistence of single-pill $A R B / C C B$ combination therapy compared to multiple-pill ARB/CCB regimens. Curr Med Res Opin 2010; 26: 2877-87.

28. Ferrario CM, Panjabi S, Buzinec P, Swindle JP. Clinical and economic outcomes associated with amlodipine/ renin-angiotensin system blocker combinations. Ther Adv Cardiovasc Dis 2013; 7: 27-39.

29. Viechtbauer W. Conducting meta analysis in $R$ with the metafor package. J Statist Software 2010; 36: 1-48.

30. Solomon LR, Dawes PM. Treatment of essential hypertension with a combination of propranolol and bendrofluazide. J Int Med Res 1980; 8: 34-7.

31. Nissinen A, Tuomilehto J. Evaluation of the antihypertensive effect of atenolol in fixed or free combination with chlorthalidone. Pharmatherapeutica 1980; 2: 462-8.

32. Olvera R, Moguel R, Meaney E, Gonzalez V, Sanchez S. Use of a combination of lisinopril and hydrochlorothiazide in the treatment of arterial-hypertension. Curr Ther Res Clin Exp 1991; 49: 155-61.

33. Mancia G, Omboni S. Candesartan plus hydrochlorothiazide fixed combination vs previous monotherapy plus diuretic in poorly controlled essential hypertensive patients. Blood Pressure 2004; 13: 11-7.

34. Bengtsson C, Schroder G, Malmberg L, Abrahamsson L Sandrstedt R. Antihypertensive treatment with diuretics and beta-blockers in free and fixed-ratio combination: a clinical trial with oxprenolol/chlorthalidone in a fixed-ratio combination. Clin Ther 1979; 2: 114-23.

35. Ebbutt AF, Elsdon-Dew RW. A multicentre study examining the substitution of Trasidrex for the free combination of Slow-Trasicor and Navidrex-K. J Int Med Res 1979; 7: 524-7.

36. Forrest WA. An open comparison between free and a fixed combination of diuretic and beta-blocker in the management of essential hypertension. J Int Med Res 1980; 8: 127-31.

37. Dezii CM. A retrospective study of persistence with single-pill combination therapy vs. concurrent two-pil therapy in patients with hypertension. Manag Care 2000; 9 (Suppl): 2-6.

38. Jackson K, Brixner D, Oderda G, Oberg B, Sheng X, Keskinaslan A. Compliance and persistence of fixed dose versus free dose combination therapy with valsartan and HCTZ for patients with hypertension [abstract]. Value Health 2006; 9: A363.

39. Hasford J, Schroder-Bernhardi D, Rottenkolber M, Kostev K, Dietlein G. Persistence with antihypertensive treatments: results of a 3-year follow-up cohort study. Eur J Clin Pharmacol 2007; 63: 1055-61.

40. Jackson KC $2^{\text {nd }}$, Sheng X, Nelson RE, Keskinaslan A, Brixner DI. Adherence with multiple-combination antihypertensive pharmacotherapies in a US managed care database. Clin Ther 2008; 30: 1558-63.

41. Barron JJ, Daniel G, Makin C, et al. Treatment modifications and resource use for fixed-dose vs separate-agent antihypertensive regimens. Drug Benefit Trends 2008; 20: 226-47.

42. Malesker MA, Hilleman DE. Comparison of amlodipine/ valsartan fixed-dose combination therapy and conventional therapy. Manag Care 2010; 19: 36-42.
43. Bronsert MR, Henderson WG, Valuck R, et al. Comparative effectiveness of antihyperthensive therapeutic classes and treatment strategies in the initiation of therapy in primary care patients: a Distributed Ambulatory Research in Therapeutics Network (DARTNet) study. J Am Board Fam Med 2013; 26: 529-38.

44. Kumagai N, Onishi K, Hoshino K, et al. Improving drug adherence using fixed combinations caused beneficial treatment outcomes and decreased health-care costs in patients with hypertension. Clin Exp Hypertens 2013; 35: 355-60.

45. Ulusoy S, Ozkan G, Konca C, Kaynar K. A comparison of the effects of fixed dose vs. single-agent combinations on 24-h blood pressure variability. Hypertens Res 2012; 35: 1111-7.

46. Kettani FZ, Dragomir A, Cote R, et al. Impact of a better adherence to antihypertensive agents on cerebrovascular disease for primary prevention. Stroke 2009; 40: 213-20.

47. Bailey JE, Wan JY, Tang J, et al. Antihypertensive medication adherence, ambulatory visits, and risk of stroke and death. J Gen Intern Med 2010; 25: 495-503.

48. Bangalore S, Kamalakkannan G, Parkar S, et al. Fixeddose combinations improve medication compliance: a meta-analysis. Am J Med 2007; 120: 713-9. 